\title{
Prospective, randomised study to compare empirical treatment versus targeted treatment on the basis of the urine antigen results in hospitalised patients with community-acquired pneumonia
}

\author{
M Falguera, ${ }^{1}$ A Ruiz-González, ${ }^{1} \mathrm{~J}$ A Schoenenberger, ${ }^{2}$ C Touzón, ${ }^{1}$ I Gázquez, \\ C Galindo, ${ }^{1} \mathrm{~J}$ M Porcel ${ }^{1}$
}

\section{See Editorial, p93}

${ }^{1}$ Internal Medicine Service, Hospital Universitari Arnau de Vilanova, Universitat de Lleida, Institut de Recerca Biomèdia de Lleida (IRBLLEIDA), Lleida, Ciber de Enfermedades Respiratorias, Spain ${ }^{2}$ Pharmacy Services, Hospital Universitari Arnau de Vilanova, Universitat de Lleida, Institut de Recerca Biomèdia de Lleida (IRBLLEIDA), Lleida, Spain

\section{Correspondence to}

Professor Miquel Falguera,

Service of Internal Medicine

Hospital Universitari Arnau de

Vilanova, Rovira Roure 80,

25198 Lleida, Spain;

mfalguera@comll.cat

Received 25 April 2009 Accepted 3 August 2009

Published Online First

23 August 2009

\begin{abstract}
Background Recommendations for diagnostic testing in hospitalised patients with community-acquired pneumonia remain controversial. The aim of the present study was to evaluate the impact of a therapeutic strategy based on the microbiological results provided by urinary antigen tests for Streptococcus pneumoniae and Legionella pneumophila.
\end{abstract}

Methods For a 2-year period, hospitalised patients with community-acquired pneumonia were randomly assigned to receive either empirical treatment, according to international guidelines, or targeted treatment, on the basis of the results from antigen tests. Outcome parameters, monetary costs and antibiotic exposure levels were compared.

Results Out of 194 enrolled patients, 177 were available for randomisation; 89 were assigned to empirical treatment and 88 were assigned to targeted treatment. Targeted treatment was associated with a slightly higher overall cost (€1657.00 vs $€ 1617.20, p=0.28)$, reduction in the incidence of adverse events ( $9 \%$ vs $18 \%, p=0.12$ ) and lower exposure to broad-spectrum antimicrobials (154.4 vs 183.3 defined daily doses per 100 patient days). No statistically significant differences in other outcome parameters were observed. Oral antibiotic treatment was started according to the results of antigen tests in 25 patients assigned to targeted treatment; these patients showed a statistically significant higher risk of clinical relapse as compared with the remaining population $(12 \%$ vs $3 \%, p=0.04)$.

Conclusions The routine implementation of urine antigen detection tests does not carry substantial outcome-related or economic benefits to hospitalised patients with community-acquired pneumonia. Narrowing the antibiotic treatment according to the urine antigen results may in fact be associated with a higher risk of clinical relapse.

\section{INTRODUCTION}

A decade ago, when blood and sputum cultures constituted the routine diagnostic approach in community-acquired pneumonia, several studies concluded that these conventional diagnostic tests did not contribute significantly to patient disease management. ${ }^{12}$

More recently, urinary antigen tests to diagnose pneumonia caused by Legionella pneumophila or Streptococcus pneumoniae have been introduced. ${ }^{3} 4$ Antigen tests have substantially increased the percentage of patients with aetiological diagnoses and, consequently, they have become one of the most highly employed diagnostic methods for community-acquired pneumonia. ${ }^{5-7}$ However, the clinical benefits derived from the routine application of antigen tests have not been fully evaluated, and the validity of a strategy of targeted antimicrobial therapy based on the results of these tests has not been established. The existence of polymicrobial infections, the possibility of false-positive antigen results, the potential benefits attributed to combination therapies in cases of severe or bacteraemic pneumonia and the inability to determine the antimicrobial susceptibility of pathogens have led to questions about the cost-effectiveness of this practice. $^{89}$

Given these unresolved issues and the lack of scientific evidence, guidelines suggest a potential value of antigen testing in certain subgroups of patients, especially those with underlying conditions or those with a more severe clinical picture; alternatively, they leave the decision in the hands of physicians according to clinical or epidemiological circumstances. ${ }^{10} 11$

The purpose of our study was to perform an overall evaluation of clinical and economic consequences derived from the routine implementation of urinary antigen tests in hospitalised patients with community-acquired pneumonia.

\section{METHODS}

\section{Study setting and patient selection}

A prospective, randomised, comparative trial was conducted from April 2006 to March 2008. We recruited study participants from the Internal Medicine Department in the Hospital Universitari Arnau de Vilanova in Lleida, Catalonia, Spain. All adult patients admitted from the Emergency Department with a diagnosis of communityacquired pneumonia were eligible to enter in the study.

Inclusion criteria were:

1. Age $\geq 18$ years.

2. Clinical and radiological evidence of pneumonia consisting of two or more of the following clinical manifestations: fever, chills, cough, sputum production, pleuritic chest pain and signs of lung consolidation; along with the presence of an infiltrate in the chest radiograph that was consistent with acute infection. 
3. Class IVor Vof the Pneumonia Severity Index or the presence of additional circumstances that justify hospital admission.

4. Clinical stability between 2 and 6 days after admission, defined as the condition in which all the following threshold values were achieved for a $24 \mathrm{~h}$ period: temperature, $\leq 37.2^{\circ} \mathrm{C}$; heart rate $\leq 100$ beats/min; respiratory rate, $\leq 24$ breaths/ min; systolic blood pressure, $\geq 90 \mathrm{~mm} \mathrm{Hg}$; and oxygen saturation of $\geq 90 \%$ or arterial oxygen partial pressure of $\geq 60 \mathrm{~mm} \mathrm{Hg}$ when the patient was not receiving supplemental oxygen. ${ }^{12}$

Exclusion criteria were:

1. Misdiagnosis at admission.

2. Nosocomial-, nursing home- or healthcare-associated pneumonia.

3. Risk factors for infection due to Pseudomonas aeruginosa, anaerobia or other microorganisms that require alternative therapeutic regimens.

4. Infection caused by tuberculosis or opportunistic microorganisms.

5. Empyema at admission.

6. Immunosuppression, for reasons including HIV infection, haematological neoplasms, solid-organ and bone-marrow transplantation, neutropenia and immunosuppressive treatments.

Patients provided written informed consent to participate in the trial. The study was approved by the scientific and ethic committees of our institution.

\section{Procedures and treatment}

Therapeutic strategy at admission was identical for all patients and based on international guidelines. ${ }^{10} 11$ Thus, patients received one of the following two intravenous regimens upon entry: (1) $\beta$-lactam (ceftriaxone, $2 \mathrm{~g}$ daily, or amoxicillin-clavulanate, $1 \mathrm{~g}$ three times daily) plus macrolide (azithromycin, $500 \mathrm{mg}$ daily) or (2) fluoroquinolone (levofloxacin, $750 \mathrm{mg}$ daily), according to the preferences of the attending physician.

Patients underwent a daily clinical assessment during their hospital stay and at least one follow-up visit took place 1 month after discharge. Patients who achieved clinical stability between 2 and 6 days after admission and could tolerate oral food were randomly assigned to one of two treatment arms in order to receive either empirical treatment (ET) or targeted treatment (TT) (figure 1), and were treated as follows:

1. Subjects assigned to the ET arm were treated according to recommendations from international guidelines. Those patients initially treated with a combination of $\beta$-lactam plus macrolide were switched to receiving a broad-spectrum oral $\beta$-lactam (amoxicillin-clavulanate, 875/125 mg three times daily or cefditoren, $400 \mathrm{mg}$ twice daily) to complete a 10 day course, plus oral macrolide (azithromycin, $500 \mathrm{mg}$ daily) to complete 5 days of treatment. Alternatively, patients who had received intravenous levofloxacin completed a course of 10 days with the same antibiotic (levofloxacin, $750 \mathrm{mg}$ daily).

2. Patients assigned to the TT arm were switched to oral amoxicillin, $1 \mathrm{~g}$ three times daily, to complete a 10 day course, if the pneumococcal urine antigen test was positive or to oral azithromycin, $500 \mathrm{mg}$ daily to complete a 5 day course, if the $L$ pneumophila urine antigen test was positive. Conversely, for patients with negative urinary antigen tests, oral treatment was the same as for patients assigned to the ET group.

In the absence of additional medical circumstances, patients were discharged between 24 and $48 \mathrm{~h}$ after switching from intravenous to oral treatment.

\section{Microbiological tests}

A microbiological study was performed upon entry, before randomisation, including the following samples:

1. Two sets of blood for conventional cultures.

2. Sputum for Gram stain and culture, when a good quality sample was available.

3. Pleural fluid, if present, for Gram stain and culture in conventional media.

4. Paired serum samples, at presentation and 4-8 weeks later, for serological studies to detect the presence of antibodies against Mycoplasma pneumoniae, Chlamydophila pneumoniae, Chlamydophila psittaci and Coxiella burnetii.

5. Urine for detection of antigens of $S$ pneumoniae or $L$ pneumophila by using a rapid test (BinaxNow test, Leti Laboratories, Barcelona, Spain)

\section{Endpoints and definitions}

The following endpoints were evaluated:

1. Outcome parameters: mortality, clinical relapse, admission to the intensive care unit, incidence of adverse events, length of hospital stay and readmission. Clinical relapse was considered to occur when clinically stable patients who were starting follow-up oral treatment regained clinical instability (temperature, $>37.2^{\circ} \mathrm{C}$; heart rate, $>100$ beats $/ \mathrm{min}$; respiratory rate, $>24$ breaths/min; systolic blood pressure, $<90 \mathrm{~mm} \mathrm{Hg}$; or oxygen saturation of $<90 \%$ or arterial oxygen partial pressure of $<60 \mathrm{~mm} \mathrm{Hg}$ on room air) and developed or worsened one or more clinical manifestations of respiratory infection, not attributable to alternative causes.
Figure 1 Randomisation of 194 hospitalised patients with community-acquired pneumonia and clinical relapses according to different therapeutic strategies.

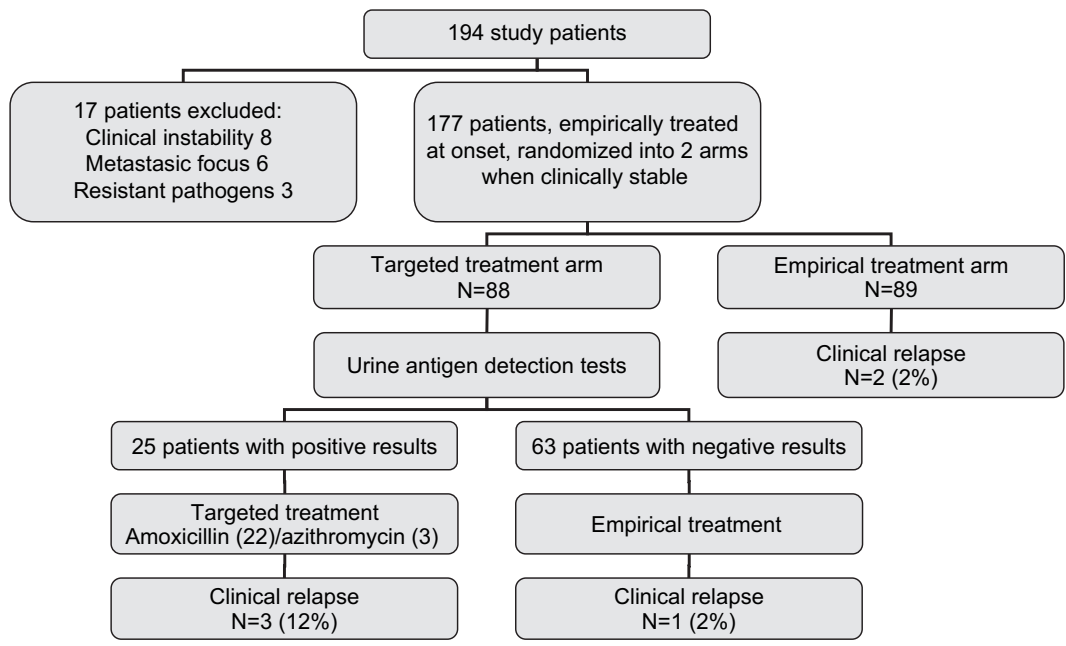


Adverse events were subjectively reported by the patients or were observed by the clinical staff during therapy and for the 30 day period posttherapy. They were defined as symptoms or signs that could not be attributed to the pneumonia, beginning after the start of antibiotic treatment.

2. Economic parameters: cost of hospital stay, cost of antimicrobial treatment and cost of diagnostic procedures. All costs were calculated in Euros.

The cost of hospital stay was calculated from the perspective of the Hospital Financial Department. Thus, costs were derived from the cost per day over the years 2005 and 2006 including room stay and basic diagnostic and therapeutic procedures (€210), multiplied by the mean number of days of hospital stay.

The daily costs of antimicrobial treatments was calculated from the perspective of the Hospital Pharmacy. The respective daily costs of antimicrobials were: $€ 1.20$ for $2 \mathrm{~g}$ of intravenous ceftriaxone; $€ 45.75$ for $750 \mathrm{mg}$ of intravenous levofloxacin; $€ 21.50$ for $500 \mathrm{mg}$ of intravenous azithromycin; $€ 0.92$ for $3 \mathrm{~g}$ of intravenous amoxicillin-clavulanate; $€ 4.08$ for $750 \mathrm{mg}$ of oral levofloxacin; $€ 7.90$ for $800 \mathrm{mg}$ of oral cefditoren; $€ 2.12$ for $2.62 \mathrm{~g}$ of oral amoxicillin-clavulanate; $€ 3.10$ for $500 \mathrm{mg}$ of oral azithromycin and $€ 0.88$ for 3 g of oral amoxicillin. Finally, the cost of microbiological tests was calculated from the perspective of the Hospital Laboratory of Microbiology. The cost of the pneumococcal antigen test was $€ 16.30$, and the cost of the Legionella antigen test was $€ 21.00$.

3. Exposure to antimicrobials: length of the overall antimicrobial treatment, length of intravenous antimicrobial treatment and exposure to broad-spectrum and narrow-spectrum antimicrobials. Exposure to individual antibiotics was measured using the number of defined daily doses (DDD) per 100 patient days. The number of DDD of each antibiotic was calculated using the total grams of individual antibiotics dispensed to study patients for each route of administration divided by the value of one DDD.

Ceftriaxone, amoxicillin-clavulanate, cefditoren and levofloxacin were considered broad-spectrum antimicrobials. Amoxicillin and azithromycin were considered narrow-spectrum antimicrobials.

\section{Statistical analysis}

First analysis of the endpoints was performed on all randomised patients distributed in the two treatment arms, according to the intent-to-treat principle. We also performed a subanalysis of patients according to the real strategy employed, by comparing empirically treated patients with patients treated according to antigen test results.

We compared continuous variables between groups by the Mann-Whitney $U$ test, and proportions between groups by the $\chi^{2}$ test or Fisher exact test. All statistical tests were two tailed, and the threshold of statistical significance was a $p$ value $<0.05$. We performed all statistical analysis with SPSS software, version 12.0 (SPSS, Chicago, Illinois, USA).

\section{RESULTS}

From a total of 194 eligible patients with community-acquired pneumonia, 17 were excluded for the following reasons: eight patients did not reach clinical stability, six developed a metastatic infectious focus (five, empyema; and one, endocarditis) and three had infection caused by resistant microorganisms (Paeruginosa was isolated from sputum in one case, and Klebsiella pneumoniae and Escherichia coli were each isolated from blood in two remaining patients). Consequently, 177 patients were finally entered and randomised; 89 were assigned to the ET arm, and 88 to the TT arm. Baseline demographic and clinical characteristics of patients are presented in table 1 .

No statistically significant differences in outcome parameters were found between groups (table 2). A greater incidence of minor adverse events was observed in the ET group (16 events (18\%) vs 8 events (9\%) in the ET and the TT arms, respectively), mainly referred to the gastrointestinal tract $(p=0.12)$. Treatment of one patient in each arm was stopped because of major adverse events potentially related to drugs (hepatitis in one patient in the ET arm, who had received levofloxacin; and leucocytoclastic vasculitis in one patient in the TT arm, who had received amoxicillin).

The mean overall cost of patient care was $€ 1657.00$ among subjects in the TT arm (hospital stay, $€ 1481.40$; consumption of antimicrobials, $€ 138.30$; and urine antigen tests, $€ 37.30)$ and $€ 1617.20$ among subjects in the ET arm (hospital stay, $€ 1470.70$; and consumption of antimicrobials, $€ 146.50)$ ( $p=0.28)$.

Table 3 details the antibiotic consumption in both subsets of patients. Exposure to intravenous antimicrobials was similar in both groups. In contrast, a small increase in the use of oral broadspectrum antimicrobials was observed for patients assigned to the ET arm.

Antigen detection tests were positive in 25 patients (25\%) assigned to the TT arm (S pneumoniae, 22; and L pneumophila, 3). As a consequence, 152 patients were in fact empirically treated. In comparative analyses between these subsets of patients (tables 4 and 5) no differences were found for most of the outcome parameters. However, patients treated according to their urine antigen results showed a higher incidence of clinical

Table 1 Intent-to-treat analysis of demographic and baseline characteristics of the study patients distributed in two arms: empirical treatment and targeted treatment

\begin{tabular}{|c|c|c|c|}
\hline Characteristics & $\begin{array}{l}\text { Empirical } \\
\text { treatment } \\
(\mathrm{n}=89)\end{array}$ & $\begin{array}{l}\text { Targeted } \\
\text { treatment } \\
(\mathrm{n}=\mathbf{8 8})\end{array}$ & p Value \\
\hline \multicolumn{4}{|l|}{ Epidemiological data } \\
\hline Age, years & $64 \pm 19.2$ & $65 \pm 20.1$ & 0.88 \\
\hline Males & $58(65)$ & $59(67)$ & 0.87 \\
\hline Smoking habit & $17(19)$ & $18(20)$ & 0.85 \\
\hline Alcohol abuse & $4(4)$ & $8(9)$ & 0.25 \\
\hline COPD & $16(18)$ & $20(23)$ & 0.46 \\
\hline Diabetes mellitus & $18(20)$ & $13(15)$ & 0.43 \\
\hline Chronic heart failure & $10(11)$ & $7(8)$ & 0.61 \\
\hline Chronic liver disease & $2(2)$ & $3(3)$ & 0.68 \\
\hline Chronic renal disease & $4(4)$ & $5(6)$ & 0.75 \\
\hline Neoplasm & $5(6)$ & $9(10)$ & 0.28 \\
\hline Prior antibiotic therapy & $21(24)$ & $18(21)$ & 0.72 \\
\hline \multicolumn{4}{|l|}{ Clinical findings } \\
\hline Heart rate, beats/min & $97 \pm 17.9$ & $101 \pm 17.2$ & 0.76 \\
\hline Respiratory rate, cycles/min & $25 \pm 8.1$ & $27 \pm 8.6$ & 0.62 \\
\hline $\mathrm{PO}_{2}$ & $64 \pm 22.1$ & $67 \pm 20.9$ & 0.70 \\
\hline \multicolumn{4}{|l|}{ Radiological features } \\
\hline Multilobar infiltrate & $15(17)$ & $12(14)$ & 0.68 \\
\hline Pleural effusion & $12(14)$ & $7(8)$ & 0.33 \\
\hline \multicolumn{4}{|l|}{ PSI categories } \\
\hline Class IV-V & $52(58)$ & $51(58)$ & 1.00 \\
\hline \multicolumn{4}{|l|}{ Initial intravenous treatment regimen } \\
\hline Ceftriaxone plus macrolide & $65(73)$ & $68(77)$ & 0.66 \\
\hline Amoxicillin-clavulanate plus macrolide & $4(4)$ & $2(2)$ & \\
\hline Levofloxacin & $20(22)$ & $18(20)$ & \\
\hline
\end{tabular}

Data are presented as mean $\pm S D$, or numbers of patients (percentage). COPD, chronic obstructive pulmonary disease; $\mathrm{PO}_{2}$, partial pressure of oxygen; PSI Pneumonia Severity Index. 
Table 2 Intent-to-treat comparative analysis of the outcomes of study patients distributed in two arms: empirical treatment and targeted treatment

\begin{tabular}{llll}
\hline Outcome parameters & $\begin{array}{l}\text { Empirical } \\
\text { treatment } \\
(\mathbf{n = 8 9 )}\end{array}$ & $\begin{array}{l}\text { Targeted } \\
\text { treatment } \\
(\mathbf{n = 8 8})\end{array}$ & $\begin{array}{l}\mathbf{p} \\
\text { Value }\end{array}$ \\
\hline Death & 0 & $1(1)$ & 0.50 \\
Clinical relapse & $2(2)$ & $4(5)$ & 0.44 \\
Admission to the intensive care unit & $1(1)$ & 0 & 1.00 \\
Length of hospital stay, days & $7.1 \pm 3.8$ & $7.1 \pm 4.0$ & 0.97 \\
Readmission & $2(2)$ & $4(5)$ & 0.44 \\
Adverse events & $16(18)$ & $8(9)$ & 0.12 \\
Length of antimicrobial treatment, days & $10.5 \pm 1.3$ & $10.8 \pm 1.6$ & 0.83 \\
Length of intravenous treatment, days & $5.0 \pm 2.6$ & $5.2 \pm 3.1$ & 0.55 \\
\hline
\end{tabular}

Data are presented as mean $\pm \mathrm{SD}$, or number of patients (percentage).

relapse, seen in 3 of 25 patients (12\%), all receiving amoxicillin. These three patients were men; 60, 61 and 91 years old, respectively; and only one had a non-severe chronic obstructive pulmonary disease as underlying co-morbidity. They initially received the combination $\beta$-lactam plus macrolide and, between 2 and 4 days after admission, when clinically stable, initiated the oral regimen with amoxicillin, $1 \mathrm{~g}$ three times daily, but were found to be clinically unstable 24-48 h later, manifested by fever and many of the symptoms that they showed at entry. Although daily monitoring of biological parameters was not performed, the three patients also showed, during relapse, high C-reactive protein levels (84.3, 107.3 and $221.5 \mathrm{mg} / \mathrm{l}$, respectively). None of these patients developed empyema, parapneumonic effusion or other metastatic foci. In all cases, the outcome was favourable only on restarting the initial empirical regimen. An amoxicillinresistant $E$ coli was later isolated from initial blood cultures in one case, but all complementary microbiological tests provided negative results for the remaining two patients. In contrast, only 3 of 152 empirically treated patients (2\%) experienced a clinical relapse with the oral regimen $(p=0.04)$. Small reductions in the incidence of adverse events, and in the use of oral broad-spectrum antimicrobials (levofloxacin, amoxicillin-clavulanate and cefditoren), mainly replaced by oral amoxicillin, were found in patients treated according to positive antigen tests.

Table 3 Intent-to-treat evaluation of exposure to antimicrobials in study patients distributed in two arms: empirical treatment and targeted treatment

\begin{tabular}{lcc}
\hline Exposure to antimicrobials & $\begin{array}{c}\text { Empirical } \\
\text { treatment } \\
(\mathbf{n = 8 9 )}\end{array}$ & $\begin{array}{c}\text { Targeted } \\
\text { treatment } \\
(\mathbf{n = 8 8})\end{array}$ \\
\hline Broad-spectrum antimicrobials & & \\
Ceftriaxone, IV & 32.8 & 38.8 \\
Levofloxacin, IV & 16.2 & 12.7 \\
Levofloxacin, oral & 23.8 & 19.4 \\
Amoxicillin-clavulanate, IV & 3.9 & 3.0 \\
Amoxicillin-clavulanate, oral & 72.7 & 49.4 \\
Cefditoren, oral & 33.9 & 31.1 \\
Total & 183.3 & 154.4 \\
Narrow-spectrum antimicrobials & & \\
Azithromycin, IV & 30.1 & 35.1 \\
Azithromycin, oral & 18.2 & 13.0 \\
Amoxicillin, oral & 0 & 50.1 \\
Total & 48.3 & 98.2 \\
\hline
\end{tabular}

Data are presented as defined daily doses per 100 patient days.

IV, intravenous.
Table 4 Comparative analysis of outcomes between subsets of patients according to the therapeutic strategy employed

\begin{tabular}{llll}
\hline & $\begin{array}{l}\text { Patients } \\
\text { empirically } \\
\text { treated } \\
\text { (n=152) }\end{array}$ & $\begin{array}{l}\text { Patients } \\
\text { treated } \\
\text { according } \\
\text { to antigen } \\
\text { results } \\
\text { (n=25) }\end{array}$ & p Value \\
\hline Outcome parameters & $1(1)$ & 0 & 1.00 \\
Death & $3(2)$ & $3(12)$ & 0.04 \\
Clinical relapse & $1(1)$ & 0 & 1.00 \\
Admission to the intensive care unit & $7.0 \pm 3.7$ & $7.2 \pm 4.2$ & 0.46 \\
Length of hospital stay, days & $4(3)$ & $3(12)$ & 0.06 \\
Readmission & $22(14)$ & $2(8)$ & 0.54 \\
Adverse events & $10.4 \pm 1.4$ & $10.8 \pm 1.9$ & 0.54 \\
Length of antimicrobial treatment, days & &
\end{tabular}

Data are presented as mean $\pm \mathrm{SD}$, or number of patients (percentage).

\section{DISCUSSION}

In this prospective, randomised study, we evaluated the overall consequences of the implementation of urine antigen detection tests in order to establish infection by $S$ pneumoniae or L pneumophila in a cohort of hospitalised patients with community-acquired pneumonia. Our results suggest that this microbiological information does not provide benefits in terms of patient outcome or cost-effectiveness. In fact, only small reductions in the incidence of adverse events and exposure to broad-spectrum antimicrobials were observed. Conversely, the narrowing of therapy on the basis of antigen test results may carry a higher risk of clinical relapse.

It has been extensively demonstrated that the implementation of pneumonia guidelines produces substantial benefit to patients. Guideline-concordant antibiotic treatment has been associated with improved in-hospital survival, shorter time to clinical stability, shorter time to switch therapies and reduced hospital stay. ${ }^{13-16}$ Therefore, the findings from our study, showing the absence of patient outcome benefits based on the use of urine antigen tests, were somewhat predictable. No benefits in terms of mortality, pneumonia-related complications and length of hospitalisation, as compared with patients treated according to guidelines, were seen.

The most remarkable finding of our study is related to the risk of treatment failure after starting oral treatment guided by

Table 5 Evaluation of exposure to antimicrobials in both subsets of patients according to the therapeutic strategy employed

\begin{tabular}{lcc}
\hline Exposure to antimicrobials & $\begin{array}{l}\text { Patients empirically } \\
\text { treated (n=152) }\end{array}$ & $\begin{array}{l}\text { Patients treated } \\
\text { according to antigen } \\
\text { results (n=25) }\end{array}$ \\
\hline Broad-spectrum antimicrobials & & \\
Ceftriaxone, IV & 35.9 & 35.2 \\
Levofloxacin, IV & 16.1 & 15.8 \\
Levofloxacin, oral & 26.1 & 0 \\
Amoxicillin-clavulanate, IV & 3.3 & 4.4 \\
Amoxicillin-clavulanate, oral & 69.6 & 10.0 \\
Cefditoren, oral & 36.7 & 4.0 \\
Total & 187.7 & 69.4 \\
Narrow-spectrum antimicrobials & & \\
Azithromycin, IV & 32.8 & 35.0 \\
Azithromycin, oral & 15.8 & 11.1 \\
Amoxicillin, oral & 0 & 176.4 \\
Total & 48.6 & 222.5
\end{tabular}

Data are presented as defined daily doses per 100 patient days.

IV, intravenous. 
antigen test results. Twelve per cent of clinically stable patients who were switched to oral amoxicillin in concordance with a positive urine antigen test showed a clinical relapse (as compared with only $2 \%$ of empirically treated subjects). In one patient, an amoxicillin-resistant $E$ coli infection was later isolated from samples of blood obtained at admission. We can only speculate as to whether this patient had a dual infection or a false-positive result of pneumococcal urine antigen test. In other cases, no microbiological causes to justify the poor outcomes were found; however, similarly, dual infections or falsepositive results from urine antigen detection tests are the most reasonable explanations. The inclusion of additional rapid tests in the initial strategy in order to detect atypical agents could reduce the risk of uncovered agents in mixed infections; however, we know that a high proportion of pathogens remain undetected in community-acquired pneumonia, even employing a wide battery of diagnostic tests. Finally, we believe that, given current levels of $S$ pneumoniae penicillin resistance, it seems more difficult to attribute treatment failure to potential amoxicillin resistance of pathogens. ${ }^{17} 18$

Hospital stay is the most important determinant of the total costs for hospitalised patients with community-acquired pneumonia. ${ }^{19-21}$ Therefore, it is unlikely that a strategy that is not associated with a reduction in the hospital stay would be able to reduce costs. In our study, which showed no statistically significant differences in the length of hospitalisation, costs of diagnostic procedures exceeded the reduction of costs for antimicrobials in patients with TT. In fact, four sets of antigen tests were needed to facilitate the use of narrow-spectrum antimicrobials in one patient, and this strategy was only applicable between 2 and 4 days after admission. Certainly, earlier introduction of pathogen-directed therapy, even within the first $24 \mathrm{~h}$ period, would increase economic benefits, but, according to our experience, this strategy is risky in patients with severe pneumonia; additionally, several studies concluded that the maintenance of macrolides in combination with $\beta$-lactams could reduce morbidity and mortality in patients with severe pneumococcal pneumonia. ${ }^{9} 22$ The relative insensitivity of the antigen detection techniques and the limitation of the method to Legionella serogroup 1 also contribute to reducing the expected benefits.

On the positive side, we only can include more questionable benefits. First, we found a non-statistically significant reduction in the incidence of adverse events, although we suspect that the difference could reach statistical significance if we increased the sample size. These adverse events were non-severe, mainly related to the gastrointestinal tract and not associated with a withdrawal of treatment. Secondly, increased use of narrowspectrum antimicrobials undoubtedly carries ecological benefits, reducing the pressure for sustained antibiotic resistance. However, in clinical practice, the real impact of this strategy appears to be very slight taking into consideration that this policy was only applicable to $25 \%$ of the patients, for approximately half of the treatment duration and mainly for a period of outpatient care. Finally, we must recognise the epidemiological value of results provided by urine antigen tests, although they are limited by the lack of information about antimicrobial susceptibilities of the aetiological agents.

Previous reports in this field are quite scarce. Guchev et al reported a non-randomised study that evaluated a therapeutic approach based on the results of the pneumococcal urinary antigen test in young immunocompetent patients with nonsevere pneumonia. ${ }^{23}$ Subjects who had a positive test were treated with amoxicillin and the remaining patients received clarithromycin. The difference in clinical success rates between groups was not statistically significant, although treatment failed for $10 \%$ of patients receiving amoxicillin (as opposed to $6 \%$ of patients receiving clarithromycin). On the other hand, van der Eerden et al published a randomised, controlled trial that compared empirical antibiotic treatment and pathogen-directed treatment. In their study, therapeutic decisions were taken on the basis of clinical presentation and microbiological results, including results of urine antigen detection tests. ${ }^{24}$ The authors were unable to associate pathogen-directed antibiotic strategy with statistically significant benefits in terms of mortality or additional outcome parameters, except for a reduction in minor adverse events. In this study, 15 of 62 patients (24\%) treated according to rapidly obtained microbial results suffered failure of their treatment.

In summary, our results suggest that the routine implementation of urine antigen tests for early detection of $S$ pneumoniae and $L$ pneumophila, pathogens with patterns of antimicrobial susceptibility covered by recommended regimens in guidelines, would not greatly benefit hospitalised patients with community-acquired pneumonia.

Funding Ciber de Enfermedades Respiratorias (Ciberes 06/06/0028) Spain.

Competing interests None.

Ethics approval This study was conducted with the approval of the Scientific and ethic committees of Hospital Universitari Arnau de Vilanova, Lleida, Spain.

Patient consent Obtained.

Provenance and peer review Not commissioned; externally peer reviewed.

\section{REFERENCES}

1. Sanyal S, Smith PR, Saha AC, et al. Initial microbiologic studies did not affect outcome in adults hospitalized with community-acquired pneumonia. Am J Respir Crit Care Med 1999;160:346-8.

2. Theerthakarai R, El-Halees W, Ismail M, et al. Nonvalue of the initial microbiological studies in the management of nonsevere community-acquired pneumonia. Chest 2001;119:181-4.

3. Domínguez J, Galí N, Blanco S, et al. Detection of Streptococcus pneumoniae antigen by a rapid immunochromatographic assay in urine samples. Chest 2001;119:243-9.

4. Waterer GW, Baselski VS, Wunderink RG. Legionella and community-acquired pneumonia: a review of current diagnostic tests from a clinician's viewpoint. Am J Med 2001;110:41-8.

5. Andreo F, Domínguez J, Ruiz J, et al. Impact of rapid urine antigen tests to determine the etiology of community-acquired pneumonia in adults. Respir Med 2006; 100:884-91.

6. Díaz A, Barria $\mathrm{P}$, Niederman $\mathrm{M}$, et al. Etiology of community-acquired pneumonia in hospitalized patients in Chile. The increasing prevalence of respiratory viruses among classic pathogens. Chest 2007;131:779-87.

7. Wattanathum A, Chaoprasong C, Nunthapisud $P$, et al. Community-acquired pneumonia in southeast Asia. The microbial differences between ambulatory and hospitalized patients. Chest 2003;123:1512-19.

8. Pesola GR. The urinary antigen tests fort he diagnosis of pneumococcal pneumonia. Chest 2001;119:9-11.

9. Waterer GW, Somes GW, Wunderink RG. Monotherapy may be suboptimal for severe bacteremic pneumococcal pneumonia. Arch Intern Med 2001;161:1837-42.

10. Mandell LA, Wunderink RG, Anzueto A, et al. Infectious Diseases Society of America/American Thoracic Society consensus guidelines on the management of community-acquired pneumonia in adults. Clin Infect Dis 2007;44:S27-72.

11. Woodhead M, Blasi F, Ewig S, et al. Guidelines for the management of adult lower respiratory tract infections. Eur Respir J 2005;26:1138-80.

12. Menéndez R, Torres A, Rodríguez de Castro F, et al. Reaching stability in community acquired pneumonia: the effects of the severity of disease, treatment, and the characteristics of patients. Clin Infect Dis 2004;39:1783-90.

13. Dambrava PG, Torres A, Vallès $X$, et al. Adherence to guidelines' empirical antibiotic recommendations and community-acquired pneumonia outcome. Eur Respir J 2008;32:892-901.

14. Shorr AF, Bodi M, Rodríguez A, et al. Impact of antibiotic guideline compliance on duration of mechanical ventilation in critically ill patients with community-acquired pneumonia. Chest 2006;130:93-100.

15. Frei CR, Restrepo MI, Mortensen EM, et al. Impact of guideline-concordant empiric antibiotic therapy in community-acquired pneumonia. Am J Med 2006;119:865-71. 
16. Dean NC, Bateman KA, Donnelly SM, et al. Improved clinical outcomes with utilization of a community-acquired pneumonia guideline. Chest 2006;130 794-9.

17. Ardanuy C, Tubau F, Pallarés R, et al. Epidemiology of invasive pneumococcal disease among adult patients in Barcelona before and after pediatric 7-valent pneumococcal conjugate vaccine introduction. 1997-2007. Clin Infect Dis 2009; 48:57-64.

18. Garau J, Twynholm M, García-Méndez E, et al. Oral pharmacokinetically enhanced co-amoxiclav 2000/125 mg, twice daily, compared with co-amoxiclav 875/125 mg three times daily, in the treatment of community-acquired pneumonia in European adults. J Antimicrob Chemother 2003:52:826-36.

19. Bartolomé $\mathbf{M}$, Almirall J, Morera J, et al. A population-based study of the costs of care for community-acquired pneumonia. Eur Respir J 2004;23:610-16.
20. Menéndez R, Reyes $S$, Martínez $R$, et al. Economic evaluation of adherence to treatment guidelines in nonintensive care pneumonia. Eur Respir J 2007;29:751-6.

21. Reyes S, Martínez R, Vallés JM, et al. Determinants of hospital costs in communityacquired pneumonia. Eur Respir J 2008:31:1061-7.

22. Metersky ML, Ma A, Houck PM, et al. Antibiotics for bacteremic pneumonia. Improved outcomes with macrolides but not fluoroquinolones. Chest 2007:131:466-73.

23. Guchev IA, Yu VL, Sinopalnikov A, et al. Management of nonsevere pneumonia in military trainees with the urinary antigen test for Streptococcus pneumoniae: an innovative approach to targeted therapy. Clin Infect Dis 2005:40:1608-16.

24. van der Eerden MM, Vlaspolder F, de Graaff CS, et al. Comparison between pathogen directed antibiotic treatment and empirical broad spectrum antibiotic treatment in patients with community-acquired pneumonia: a prospective randomised study. Thorax 2005;60:672-8.

\section{Lung alert}

\section{Seasonal predictive factors of acute respiratory tract infections in children}

Acute respiratory tract infections (ARTI) in children under 5 years of age are a leading cause of mortality worldwide, and predictive tools are invaluable in preventing infection and timely vaccination. Owing to the seasonal nature of ARTI, this German study aimed to determine the effect of climate on ARTI in children requiring hospitalisation.

This retrospective study investigated the association between common pathogens in children and various meteorological parameters. Between 2003 and 2006, nasopharyngeal aspirates were taken from 2012 children ( $<16$ years) admitted to hospital with ARTI. These were analysed for 19 pathogens. Data on climate taken from the University of Mainz were averaged over 14 days and included measurement of temperature, relative humidity, wind speed and atmospheric pressure.

At least one ARTI pathogen was identified in $66.9 \%$ of samples, the most common being rhinovirus, respiratory syncytial virus (RSV), adenovirus and influenza A. Influenza A, RSV and adenovirus were associated with temperature, and rhinovirus with humidity. In addition, RSV, influenza A and human metapneumovirus showed winter peaks. In a time series model, only RSV was found to be associated with hospitalisation for ARTI in children.

This retrospective study provides intriguing data into the prediction of seasonal respiratory tract infection in children. Application of seasonal patterns to pathogen prevalence may be helpful in the management of other respiratory diseases.

- du Prel J-B, Puppe W, Gröndahl B, et al. Are meteorological parameters associated with acute respiratory tract infections? Clin Infect Dis 2009;49:861-8.

\section{Shelley A Srivastava}

Respiratory Medicine, St George's Hospital, London, UK

Correspondence to Dr Shelley A Srivastava, SpR, Respiratory Medicine, St George's Hospital, London, UK; ssrivast@sgul.ac.uk

Competing interests None.

Provenance and peer review Not commissioned; not externally peer reviewed.

Thorax 2010;65:106. doi:10.1136/thx.2009.132878 